\title{
Regional Analysis on Chinese Internet Ads with Click-Through Rates
}

\author{
Wei Li Qiang Wei Yaoqiang Chen \\ School of Economics and Management, Tsinghua University, Beijing 100084, China \\ Email: \{liw.09,weiq, chenyq.03\}@sem.tsinghua.edu.cn
}

\begin{abstract}
Internet Ads is one of the important forms of E-business activities, due to its high targeting, rich contents, flexible media as well as high interactivity. Since the economy and Internet development of different regions in China are quite differentiated, the Internet Ads of different regions have significant regional characteristics. Based on the real log data from a Chinese Internet Ads intermediate, this paper empirically analyzes the differences and characteristics of Internet Ads of China's regions with the Click-Through Rates. Moreover, this paper proposes some suggestions for decision supporting Chinese Internet Ads players, i.e., websites, intermediates and advertisers, from a managerial perspective.
\end{abstract}

Keywords: E-business, Internet Ads, Click-Through Rate, Regional Analysis

\section{Introduction}

Internet Ads is one of the important forms of e-business activities, due to its high targeting, rich contents, flexible media as well as high interactivity, which is keeping pace with four major traditional media ads, such as Television Ads, Radio Ads, Newspaper Ads and Magazine Ads [1]. According to industry reports, in 2009, even with financial crisis, Chinese Internet Ads market is still booming, reaching 20.73 billion RMBs, continuing rapid growth of $21.9 \%$ [2]. Since 2003,
Internet Ads market has continued highspeed growth of $50 \%$ roughly, occupying the total advertising market share from $3.8 \%$ in 2006 to over $10 \%$ in 2009 [2]. Nowadays, Internet Ads is going to show its unique advantages and expand the market share significantly.

Although Internet Ads market is expanding rapidly, the effect of Internet Ads is not satisfactory, since Internet users become to more and more immune from Internet Ads. Furthermore, users frequently complain the inconvenience brought by Internet Ads. One evidence is that the Click-Through Rates (CTRs) of Internet Ads decline significantly, e.g., from $30 \% \sim 50 \%$ on Banner Ads in middle 1990 s to less than $0.1 \%$ currently [3].

CTR is actually one of the key measures to evaluate the performance of Internet Ads, which is impacted by many factors, e.g., visibility of Ads, attitudes of users, interactive environment, etc $[4,5,6]$. Besides, region is also one of the important factors which show some interesting relationship with CTRs, e.g., the Internet behaviors of people in different regions will show different patterns on browsing/clicking Ads, leading to different CTRs. This paper is to empirically analyze the difference, characteristics of Internet Ads of China's different regions with CTRs data from some real log data of a Chinese Internet Ads intermediate.

The paper is organized as follows. Section 1 will investigate the relationship between regions and CTRs. The data description and methodology used in this 
paper will be presented in Section 3. The research questions and hypotheses will be proposed in Section 4 with some empirical analysis. Section 5 will briefly evaluate the results and present some countermeasures for Internet players from a managerial perspective.

\section{Regions and Internet Ads' CTRs}

Internet Ads is one kind of information dissemination activities for advertisers paying to persuade the public to buy products, aimed at influencing people's attitude to products or services in the advertisement so that advertisers can make profit. Therefore, the effects of Internet Ads, e.g., CTRs, are closely paid attention to by Internet Ads players, i.e., websites, intermediates and advertisers.

The factors that influence CTRs can be classified into three general categories: the advertisement features [4], which are the attributes and features of Internet Ads themselves, such as color, size, animation, sound, scenes, interactive and virtual characters, etc.; consumer-related factors [5], which are attitudes of users, such as personal knowledge structure, personal attitudes to Internet Ads; and situationrelated factors [6], which are Internet Ads environments, such as the relevance between Internet Ads and web pages' contents, the location of Internet Ads, etc. Nevertheless, among the researches on factors impacting CTRs of Internet Ads, there is few talking about region factors. Chinese Silly Catalog concept proposed that regionalization is of great importance to Internet services [7], especially in China. $\mathrm{Li}$ and Wei indicated that different regions could create different and individual distinctive marketing styles due to different regional cultures in China, which also took significant impacts on Internet Ads. Thereafter, regions should be taken into consideration while analyz- ing the performance, e.g., CTRs, of Internet Ads.

Besides, due to the imbalanced regional development of Internet in China, region factor plays a specifically important role in China. Moreover, due to the lack of real $\log$ data of Internet Ads, empirical studies could seldom be conducted.

\section{Data and Methodology}

In this research, a real log data of Internet Ads was provided by a professional Internet Ads intermediate in China, which provides a platform with typical Internet Ads services, such as posting, monitoring, performance evaluation and optimization, etc. There are more that 100 allied websites on the platform with thousands of Internet Ads provided by hundreds of advertisers. The Internet Ads on this platform almost cover all types of industries. The users of the platform are from different regions in China. The intermediate charges advertisers with CPC. On this platform, CTR is regarded as one of the most important index for evaluating Internet Ads.

Totally, we have collected 27 days' data (March 30 - April 26, 2009) with overall 500GB data including displaying data and clicking data. MySQL was used for data cleaning and pre-processing. Since the data from Hong Kong, Macao, Taiwan are quite limited, thus we focus on the 31 regions/provinces in Chinese mainland.

After pre-processing, the generated CTRs of each province are listed in Table 1.

\begin{tabular}{lllll}
\hline Day & Shanghai & Beijing & $\ldots$ & Yunnan \\
\hline $\mathbf{1}$ & 3.00678 & 3.63746 & $\ldots$ & 7.07463 \\
\hline $\mathbf{2}$ & 3.53536 & 3.33896 & $\ldots$ & 5.50929 \\
\hline$\ldots$ & $\ldots \ldots$ & $\ldots \ldots$ & $\ldots$ & $\ldots \ldots$ \\
\hline $\mathbf{2 6}$ & 4.15278 & 3.85921 & $\ldots$ & 6.85270 \\
\hline
\end{tabular}

Table 1: Regional Average CTRs $\left(1 / 10^{4}\right)$. 
Since no evidence shows that the data following normal distribution, this paper adopts the non-parametric test methods, i.e., Friedman test method and Wilcoxon test method. SPSS statistical software was used.

\section{Hypotheses and Analysis}

First of all, it is necessary to test whether the CTRs of provincial regions are significantly different. Then the first hypothesis 1 could be proposed.

\section{$\mathrm{H}_{1}$ : Different provinces have signifi- cantly different CTRs.}

The test results of 31 provincial regions are shown in Table 2. The result show that the p-value is less than 0.05 , indicating the hypothesis could be accepted, meaning that at least two provincial regions have significant difference on CTRs.

\begin{tabular}{cccc}
\hline N & Chi-square & Df & Asymp. Sig. \\
\hline 26 & 564.010 & 30 & .000 \\
\hline
\end{tabular}

Table 2: Friedman Test of 31 provinces.

Moreover, CTRs varies in different regions, as shown in Figure 1.

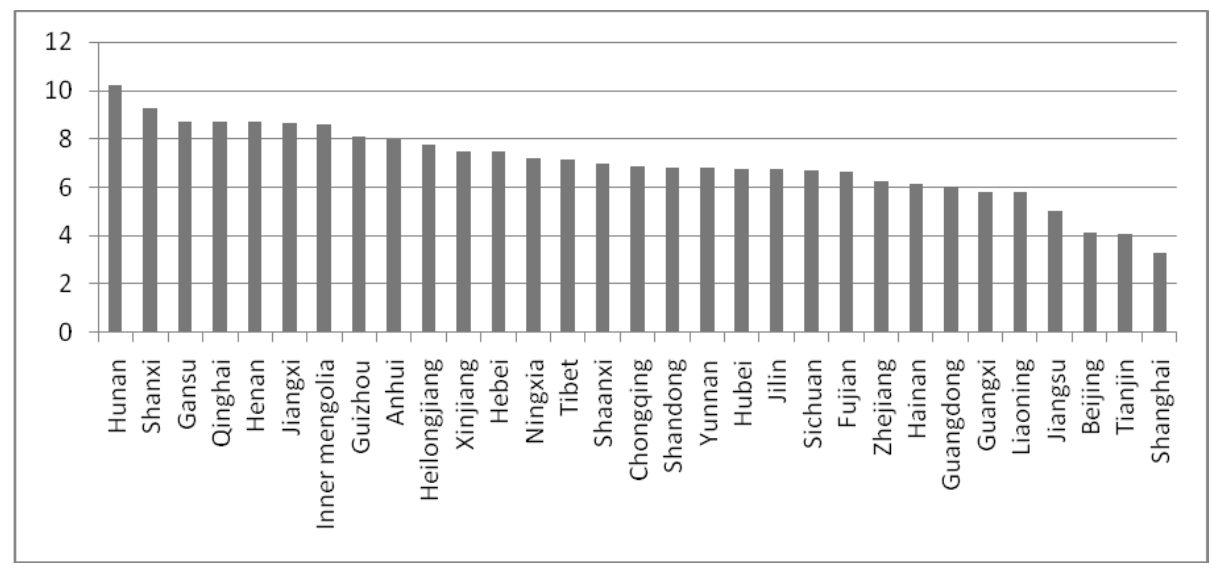

Fig. 1: CTRs of 31 provincial regions in China $\left({ }^{*} 10^{-4}\right)$.

Roughly, it could be found that the western regions, e.g., Qinghai, Tibet, etc., show higher CTRs over the eastern regions, e.g., Shanghai, Beijing, Tianjin, Jiangsu, etc.

To further validate this phenomenon, we grouped provinces into several regions, i.e., eastern region, central region, and western region (as shown in Table 3), following the official criteria [9].

Thus, to further evaluate the differences among different regions in China, a new hypothesis is proposed.

\section{$\mathrm{H}_{2}$ : Different regions have significantly different CTRs.}

The Friedman test results are shown in Table 4, which indicates that hypothesis 2 could be accepted, since p-value is less than 0.05 , meaning at least two regions have significantly different CTRs.

\begin{tabular}{lll}
\hline Eastern & Central & Western \\
\hline Beijing & Shanxi & Guizhou \\
\hline Tianjing & Jilin & Qinghai \\
\hline Shanghai & Heilongjiang & Sichuan \\
\hline Jiangsu & Anhui & Ningxia \\
\hline Guangdong & Jiangxi & Shaanxi \\
\hline
\end{tabular}




\begin{tabular}{lll}
\hline Hebei & Henan & Gansu \\
\hline Liaoning & Hubei & Inner Mongolia \\
\hline Zhejiang & Hunan & Guangxi \\
\hline Fujian & & Yunnan \\
\hline Shandong & Chongqing \\
\hline Hainan & Xinjiang \\
\hline & Tibet \\
\hline
\end{tabular}

Table 3: Classification of China's Regions.

\begin{tabular}{cccc}
\hline N & Chi-square & Df & Asymp. Sig. \\
\hline 26 & 48.308 & 2 & .000 \\
\hline
\end{tabular}

Table 4: Friedman test of 3 regions.

Moreover, to further analyze the regional difference on CTRs in details. The Wilcoxon test is performed on paired comparison on any two regions. The results are as shown in Table 5.

\begin{tabular}{ccc}
\hline & $\mathbf{Z}$ & Asymp. Sig. (2-tailed) \\
\hline $\begin{array}{c}\text { Central - } \\
\text { Eastern }\end{array}$ & $\begin{array}{c}-4.457 \\
\text { (a) }\end{array}$ & .000 \\
\hline $\begin{array}{c}\text { Central - } \\
\text { Western }\end{array}$ & $\begin{array}{c}-4.457 \\
\text { (a) }\end{array}$ & .000 \\
\hline $\begin{array}{c}\text { Western } \\
- \text { Eastern }\end{array}$ & $\begin{array}{c}-4.254 \\
\text { (b) }\end{array}$ & .000 \\
\hline
\end{tabular}

Table 5: Wilcoxon test on 3 regions.

Table 5 shows that the CTRs of any two regions are significantly different. Specifically, Central region has the highest average CTR $\left(8.26081 * 10^{-4}\right)$, Western region has the middle average CTR $\left(7.428064 * 10^{-4}\right)$, while Eastern region has the lowest average CTR (5.610809* $10^{-4}$ ), showing Eastern region has clear difference on CTRs with the other two regions. Intuitively, it shows that people in Central and Western regions of China tend to click more on Internet Ads than the people in Eastern region of China.

The major reasons that cause this situation may stem from the maturity of adopting Internet Ads, which can be further discussed in three aspects.
First, the differences of economic levels of different regions in China lead to different activeness of Internet business. For example, Eastern region in China include the most productive local economics, e.g., Shanghai, Beijing, Fujian, Guangdong, etc., while Central and Western regions do not. Higher economic level means more Internet business, leading to more Internet Ads to be displayed to the Internet users.

Second, the network penetration rates of different regions are also quite different, as shown in Figure 2. Normally, the Eastern region has the highest penetration rate, which means that Internet users are more mature and are immune to Internet Ads to some extent. Nevertheless, due to the low network penetration rates in Central and Western regions, the Internet users in those regions are still not quite antipathy to Internet Ads, which leads to higher CTRs than in Eastern region.

Third, different regions have different regional cultures, which can also take effect on the browsing and clicking behaviors on Internet Ads. Generally speaking, the people in Eastern region have more intense pace of life than Central and Western region, which means that the Internet users in Eastern region tend to retrieve useful information directly and ignore Internet Ads in many situations. However, the Internet users in Central and Western regions prefer more entertainment activities on Internet, thereafter Internet Ads, which are usually colorful and appealing, may have higher possibility to be browsed and clicked for entertainment purposes by Internet users in Central and Western regions.

To summarize the above discussions, the Internet users in Eastern region of China are more immune to Internet Ads than those in Central and Western regions of China. 


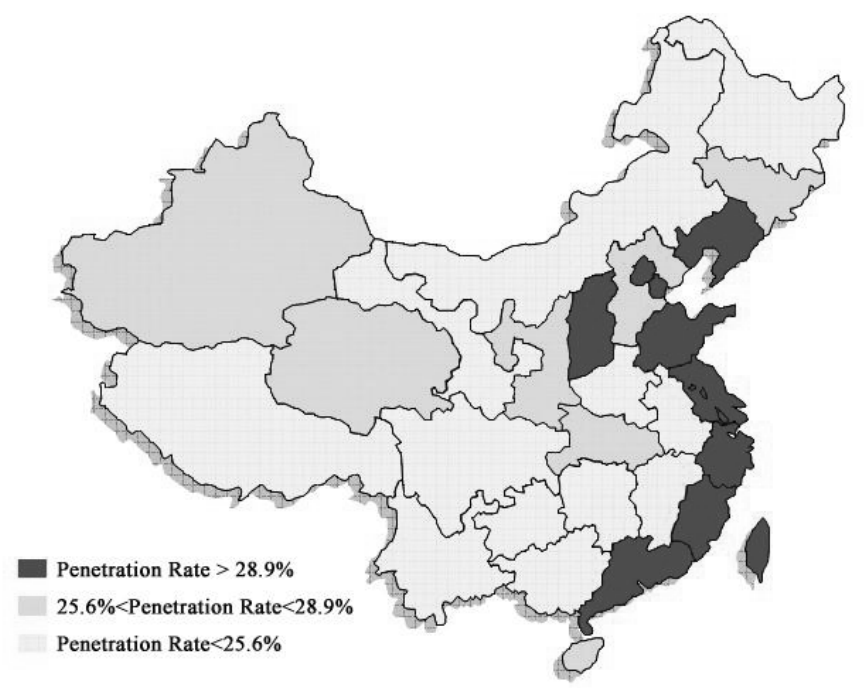

Fig. 2: Network Penetration of China ${ }^{[1]}$.

\section{Managerial Analysis}

From a managerial perspective, this section will further discuss the Internet Ads marketing strategies and countermeasures, considering the regional impacts, for three major Internet Ads players, i.e., websites as the network media, intermediates who buy advertising positions in mass and sell to advertiser separately; and the advertisers, who distribute their own Internet Ads.

For websites, if CPM (cost per thousand impressions) pricing strategy is adopted, website operators could think about to increase the CPM price of Internet Ads distributed to Central and Western region, since the number of clicks of a thousand impressions could be higher than the same Internet Ads distributed to Eastern region, due to the higher CTR, which means the click performance the Internet Ads could be better. If CPC (cost per click) pricing strategy is adopted by a website, the website operator can think about to increase the CPC price of Internet Ads distributed to Eastern region, since more impressions for per click could be generated than the same Internet Ads distributed to Central and Western regions, due to the lower CTR, meaning more impressions have been shown to Internet users in Eastern region of China.

For intermediates, generally, Internet Ads intermediates make profits by purchasing advertising positions from websites and selling them to advertisers with packaging and providing value-added Ads services. Usually, intermediates will buy some advertising position from websites will a packaged price per month. Thereafter, considering the regional impacts on CTRs, intermediates may prefer to charge the Internet Ads distributed to Central and Western regions by $\mathrm{CPC}$ pricing strategy, while charging the Internet Ads distributed to Eastern region by CPM pricing strategy, if possible, to maximize the possible arbitrage.

For advertisers, due to the high immunity of Internet Ads in Eastern region, advertisers may prefer to pay with $\mathrm{CPC}$ strategy, since low cost for clicks could be paid while high impressions could be achieved due to the lower CTR in Eastern region. In Central and Western regions, CPM could be more preferred by adver- 
tisers, since the cost for thousand impressions could be fixed, while high clicks could be achieved, due to the higher CTR in Central and Western regions.

\section{Conclusion}

Based on the 26-days logo data of from a Chinese Internet Ads intermediate, this paper discussed the regional impact on CTRs of Internet Ads in China. The empirical analysis shows that there exist significant differences among the 31 provincial regions in Chinese mainland. Moreover, the test results show that the average CTR in Eastern region in China is significantly lower than those in Central and Western regions in China. This paper also investigated the reasons that caused this situation in China, e.g., the difference of economics development, the different network penetration rates, as well as the differences in regional cultures, of Eastern, Central and Western regions of China. Thereafter, immunity of Internet Ads leads to the lower CTR like in Eastern region in China. Furthermore, based on the analysis, from a managerial perspective, some comments and countermeasure, regarding the region factor, are proposed for the three major Internet Ads players, i.e., websites, intermediates and advertisers.

There still exist some limitations in this paper. First, the log data is just from one intermediate. To what extent the data can represent the overall situation of Internet Ads in China can hardly been validated. Moreover, there exist many other factors influencing CTRs, which have also been proposed in this paper and need to be further analyzed together with region factor.

\section{Acknowledgements}

This work was partly support by the National Natural Science Foundation of China (Grant 70890083/71072015), the MOE Project of Key Research Institute of Humanities and Social Sciences at University of China (7JJD63005).

\section{References}

[1] CNNIC, The 25th Chinese Internet Development Statistic Report, 2010

[2] iResearch, Chinese Internet Ads Development Report in 2009 and 2010, 2010

[3] Gian M. Fulgoni, Marie Pauline Mörn. How online advertising works, Empirical Generalizations in Advertising Conference for Industry and Academia, December 4-5, 2008

[4] Cho, Chang-Hoan. Factors Influencing Clicking of Banner Ads on the www. Cyberpsychology \& Behavior, 2003, Vol 6, No 2, 201-215.

[5] Chang-Hoan, Hongsik John Cheon (2004). Why Do People Avoid Advertising on the Internet, Journal of Advertising, 33 (4), 89-97.

[6] Cho, Chang-Hoan. (2003). Factors Influencing Clicking of Banner Ads on the WWW. Cyberpsychology \& Behavior, 6(2), 201-215.

[7] Hong C. Silly Catalog promote Internet Localization Process. China dayily Forum, 2008

[8] Xinwei L., Zhong W. Research on Regional Internet Marketing Features among Beijing, Shanghai, Guangdong. Shanghai Information, 2009.

[9] Chun Lin, Hans Hendrischke, The Territories of the People's Republic of China. Europa Publications, First edition, 2002, London. 264 pages. 